\title{
Non-invasive dynamic near-infrared imaging and quantification of vascular leakage in vivo
}

\author{
Steven T. Proulx • Paola Luciani • Annamari Alitalo • Viviane Mumprecht • \\ Ailsa J. Christiansen - Reto Huggenberger · Jean-Christophe Leroux • \\ Michael Detmar
}

Received: 25 July 2012/ Accepted: 3 January 2013/Published online: 17 January 2013

(C) Springer Science+Business Media Dordrecht 2013

\begin{abstract}
Preclinical vascular research has been hindered by a lack of methods that can sensitively image and quantify vascular perfusion and leakage in vivo. In this study, we have developed dynamic near-infrared imaging methods to repeatedly visualize and quantify vascular leakage in mouse skin in vivo, and we have applied these methods to transgenic mice with overexpression of vascular endothelial growth factors VEGF-A or -C. Near-infrared dye conjugates were developed to identify a suitable vascular tracer that had a prolonged circulation lifetime and slow leakage into normal tissue after intravenous injection. Dynamic simultaneous imaging of ear skin and a large blood vessel in the leg enabled determination of the intravascular signal (blood volume fraction) from the tissue signal shortly after injection and quantifications of vascular leakage into the extravascular tissue over time. This method allowed for the sensitive detection of increased blood vascularity and leakage rates in K14VEGF-A transgenic mice and also reliably measured inflammation-induced changes of vascularity and leakage
\end{abstract}

Steven T. Proulx and Paola Luciani contributed equally to this work.

Electronic supplementary material The online version of this article (doi:10.1007/s10456-013-9332-2) contains supplementary material, which is available to authorized users.

S. T. Proulx - A. Alitalo - V. Mumprecht .

A. J. Christiansen · R. Huggenberger · M. Detmar ( $\square)$ Institute of Pharmaceutical Sciences, Swiss Federal Institute of Technology, ETH Zurich, Wolfgang-Pauli-Str. 10, HCI H303, 8093 Zurich, Switzerland

e-mail: michael.detmar@pharma.ethz.ch

P. Luciani $\cdot$ J.-C. Leroux $(\square)$

Institute of Pharmaceutical Sciences, Swiss Federal Institute of Technology, ETH Zurich, Wolfgang-Pauli-Str. 10, HCI H301, 8093 Zurich, Switzerland

e-mail: jean-christophe.leroux@pharma.ethz.ch over time in the same mice. Measurements after injection of recombinant VEGF-A surprisingly revealed increased blood vascular leakage and lymphatic clearance in K14-VEGF-C transgenic mice which have an expanded cutaneous lymphatic vessel network, potentially indicating unanticipated effects of lymphatic drainage on vascular leakage. Increased vascular leakage was also detected in subcutaneous tumors, confirming that the method can also be applied to deeper tissues. This new imaging method might facilitate longitudinal investigations of the in vivo effects of drug candidates, including angiogenesis inhibitors, in preclinical disease models.

Keywords Vascular permeability - Inflammation . Imaging $\cdot$ VEGF-A $\cdot$ VEGF-C

\section{Introduction}

Leakage from the bloodstream to the interstitial tissue is a tightly regulated process under normal physiological conditions. The endothelial barrier, comprised of endothelial cells and associated structures such as the glycocalyx, pericytes, and basement membrane components, controls the rate of leakage of water and plasma proteins to maintain tissue homeostasis and to provide nutrients to cells [1]. In steady state conditions there exists a slow filtration of fluid and solutes from the capillaries to the tissue that is returned to the circulation by the lymphatic system [2]. Under pathological conditions such as inflammation, this balance is disrupted leading to an influx of fluid and inflammatory cells that results in tissue swelling or edema [3]. While most inflammatory insults are acute in nature and resolve over time, chronic inflammation such as the autoimmune disorders psoriasis and rheumatoid arthritis can result in painful swelling and tissue damage. 
A major factor leading to increased plasma leakage in chronic inflammatory states is hyperpermeability of the blood vessels, which is also a characteristic feature of other disorders such as cancer and age-related macular degeneration [4]. A central mediator of vascular permeability is vascular endothelial growth factor-A (VEGF-A). VEGF-A exerts its effects acutely by vasodilation and by increasing the gaps between endothelial cells and/or by inducing formation of vesicular-vacuolar organelles in the cytoplasm [4, 5]. Chronic exposure to VEGF-A leads to an expansion of the existing vessel network by increasing the caliber of blood vessels and inducing the formation of new blood vessels. The newly formed immature angiogenic blood vessels further exacerbate chronic hyperpermeability as they typically have incomplete pericyte and basement membrane coverage.

Despite the importance of vascular hyperpermeability in many pathological conditions, methods to accurately measure vascular leakage in vivo in a non-invasive manner are limited. Traditionally, the Miles assay has been used to measure vascular leakage in skin and other organs [6]. This assay involves an intravenous injection of Evans Blue dye that binds to serum proteins (predominantly albumin) and leaks from hyperpermeable vessels that are induced by injections of acute permeability factors such as VEGF-A into the skin [7]. Quantification of the leakage requires sacrificing the animals and extracting the dye, a timeconsuming process that excludes longitudinal monitoring or further analysis of the tissue. Furthermore, the assay in its original form cannot distinguish between dye that is still within blood vessels and that which has leaked [8], complicating analyses in cases of increased vascular remodeling. Attempts to address this issue, either with perfusion to remove intravascular dye or with a two-tracer method that involves injections at different time points to differentiate the intravascular from the extravascular signals, have not gained widespread acceptance due to their experimental complexity [7, 9].

Recently, in vivo imaging approaches have been developed that allow real-time visualization of vascular leakage [10]. Dynamic contrast enhanced magnetic resonance imaging, the most established of these techniques, has advantages of increased imaging depth and good sensitivity of contrast agents, but has a limitation of poor resolution, allowing direct visualization of only the larger blood vessels [11, 12]. In addition, the complex models required for estimation of vascular permeability and the expense of equipment limit its use to dedicated imaging laboratories [9]. More recently, in vivo microscopy with fluorescent tracers, such as FITC-dextran or rhodaminealbumin conjugates, have been used to visualize vascular leakage with high resolution in a dynamic manner [13, 14]. However, these methods typically involve special experimental preparations such as dorsal skin-fold chambers or exteriorization of tissue for visualization of a limited region of interest.

We hypothesized that a dynamic fluorescence approach based on near-infrared (NIR) wavelengths could be used to visualize vascular leakage in real time in intact mouse tissues. NIR fluorescence imaging has gained popularity due to its non-invasive nature and low cost instrumentation [15]. The use of NIR wavelengths $(650-900 \mathrm{~nm})$ greatly enhances tissue penetration and detection of signals due to decreased tissue absorption and autofluorescence when compared to the visible region of the electromagnetic spectrum. Indocyanine green (ICG) is the only currently clinically approved NIR dye. However, its short half life in circulation may make it difficult to use in studies of vascular leakage [16]. Tracers that show more stable fluorescent signals in the blood would improve the visualization of leakage and allow quantifications to be developed. Such methods could be applied to an array of mouse models and allow long-term monitoring to test novel drug candidates.

In this study, we have developed NIR tracers and a simple, reliable and rapid imaging protocol for visualizing and quantifying vascular leakage in mouse tissues. We used this method to measure vascular perfusion and leakage in transgenic mice with overexpression of VEGF-A and $-\mathrm{C}$ in the skin and after local or systemic injections of VEGF-A or -C. Additionally, measurement of leakage was possible over the entire time course of chronic psoriasislike skin inflammation and in mice bearing 4T1 tumors.

\section{Materials and methods}

Transgenic mouse models

The generation of K14-VEGF-A transgenic mice that express mouse VEGF-A164 under control of the K14 promoter and of K14-VEGF-C transgenic mice that express full-length human VEGF-C have been described previously [17-19]. K14-VEGF-A and K14-VEGF-C mice were bred and housed in the animal facility of ETH Zurich. FVB wildtype mice were used as controls. All experiments were initiated when the mice were between 8 and 10 weeks of age. Mice were fed alfalfa-free chow (Provimi Klibi AG, Kaiseraugst, Switzerland) for at least 5 days prior to imaging to reduce autofluorescence signals. Experiments were performed in accordance with animal protocols approved by the Veterinäramt Kanton Zurich.

Synthesis of PEG-IRDye

Methoxypoly(ethylene glycol) (PEG) amine P20 or P40 (20 or $40 \mathrm{kDa}$, respectively, $75 \mathrm{nmol}$ ) was dissolved in 
anhydrous DMSO. The monofunctional activated polymer was labelled at room temperature for $6 \mathrm{~h}$ with $75 \mathrm{nmol}$ IRDye $^{\circledR}$ 800CW NHS Ester or IRDye ${ }^{\circledR}$ 680LT NHS Ester (D800 or D680, respectively, from LI-COR Biosciences, Lincoln, NE) predissolved in DMSO. The crude reaction was diluted in double-distilled water and lyophilized overnight. The freeze-dried mixture was reconstituted with $250 \mu \mathrm{L}$ of HEPES buffered saline (HBS, HEPES $20 \mathrm{mmol} /$ L, NaCl $142 \mathrm{mmol} / \mathrm{L}, \mathrm{pH}$ 7.4) and purified using Fluorescent Dye Removal columns (Thermo Fisher Scientific, Rockford, IL). The purity of the recovered compound (P20D680, P20-D800, P40-D680 or P40-D800) was controlled by high-performance liquid chromatography, optimizing a protocol previously described [20] as detailed in the Electronic supplementary material. The efficiency of the coupling procedure was assessed spectrophotometrically in methanol on a Cary 300 Bio UV-spectrophotometer (Agilent Technologies, Santa Clara, CA).

\section{Pharmacokinetics (PK) study}

Following tail vein injection of $0.05 \mathrm{nmol} / \mathrm{g}$ mouse weight (expressed in terms of D800 content) of P20-D800 or P40D800 into wildtype 8 week-old mice $(n=3)$, serial blood sampling from the saphenous vein $(\sim 30 \mu \mathrm{L})$ was performed at $\mathrm{t}=2 \mathrm{~min}, 15 \mathrm{~min}, 1 \mathrm{~h}$ and $3 \mathrm{~h}$ for P20-D800 and $\mathrm{t}=2 \mathrm{~min}, 15 \mathrm{~min}, 1 \mathrm{~h}, 3 \mathrm{~h}$ and $6 \mathrm{~h}$ for P40-D800. A final blood sample was collected under terminal anaesthesia by cardiac puncture $6 \mathrm{~h}$ and $12 \mathrm{~h}$ post-injection for $\mathrm{P} 20$ D800 and P40-D800, respectively. Urine was also collected at the final time point. Blood samples were left to stand for $30 \mathrm{~min}$ and then were centrifuged at $3,000 \times \mathrm{g}$ for $10 \mathrm{~min}$. Serum was collected and frozen at $-20{ }^{\circ} \mathrm{C}$ before analysis. PK parameters were determined with a serum concentration time profile. Serum was deproteinized following a protocol previously optimized [21], as detailed in the Electronic supplementary material. To investigate the presence of unbound dye at the end of the kinetics, samples $(\mathrm{t}=6 \mathrm{~h})$ from animals injected with P20-D800 or P40D800 were analysed also via high-performance liquid chromatography (HPLC). First serum was deproteinized, and afterwards eluted in an analytical HPLC. P20-D800 and pure D800 dye were also eluted as controls.

\section{Dynamic NIR imaging of vascular leakage}

Mice were anesthetized with $2 \%$ isoflurane and hair was carefully removed in the region of the saphenous vein using a razor and depilation cream at least 4 days prior to imaging. During imaging, $2 \%$ isoflurane was used for anesthesia and the mice were positioned in an IVIS Spectrum imaging system (Caliper Life Sciences, Hopkington, MA). A custom imaging platform and surgical tape were employed to ensure reproducible positioning with the tissues of interest and the saphenous vein region in the same imaging plane. A sequence of $3 \mathrm{~s}$ exposure images $\left(\lambda_{\mathrm{ex}}\right.$ : $745 \mathrm{~nm}, \lambda_{\mathrm{em}}: 800 \mathrm{~nm}$, binning of 4) was initiated and paused after the first image was acquired. Using a $30 \mathrm{~g}$ insulin syringe, $0.05 \mathrm{nmol} / \mathrm{g}$ mouse weight (expressed in terms of dye content) of PEG-IRDye solution was injected intravenously into the tail vein and the sequence was immediately resumed. Images were acquired every $15 \mathrm{~s}$ for a total imaging time of $15-30 \mathrm{~min}$ depending on the experiment. For experiments with $0.15 \mathrm{nmol} / \mathrm{g}$ mouse weight of ICG dye solution, the images were acquired with binning of 8 and exposure time of $5 \mathrm{~s}$. Using Living Image 4.0 software (Caliper), regions of interest over the tissues of interest and the saphenous vein were drawn and signal intensity values at each time point in fluorescent counts were determined. The data were exported for analysis as described in the Electronic supplementary material.

Acute permeability studies

Human VEGF-A165, VEGF-C, and VEGF-C156S were purchased from R\&D Systems (Minneapolis, MN). For examination of the effects of these proteins on vascular leakage, a modified Miles Assay was developed and performed in wildtype mice and in K14-VEGF-C (females, 10 weeks age, $n=5$ each) mice. Briefly, mice were injected with a $30 \mathrm{~g}$ syringe intradermally in one ear with $3 \mu \mathrm{L}$ solutions of $30 \mathrm{ng}$ of VEGF-A or VEGF-C and the contralateral ear with PBS as a control. The mice were immediately positioned in the IVIS and the imaging sequence as above was initiated. Tail vein injections of the imaging conjugates were performed on average 3 min after the ear injections. Additionally, mice were examined for vascular leakage in the ears after a tail vein injection of $1 \mu \mathrm{g}$ of recombinant VEGF-A protein in solution along with the dye conjugates. As systemic injection of VEGF-A caused a detectable increase in blood vessel signal at early time points, consistent with a vascular dilation effect, the vascular leakage was monitored at the time point starting at which the fluorescence enhancement reached a maximum, which was typically around 2-3 min after injection.

Miles permeability assay

A solution of $0.2 \mathrm{~mL}$ of $0.5 \%$ Evan's blue dye (Sigma) was administered by tail vein injection in wildtype mice $(n=5)$. Immediately after this injection, a $3 \mu \mathrm{L}$ solution of $30 \mathrm{ng}$ of VEGF-A was injected into the right ear while the left ear was injected with PBS as a control. After $30 \mathrm{~min}$, the ears of the mice were photographed and then harvested from the sacrificed animals. The ears were split 
using forceps and placed in $0.5 \mathrm{~mL}$ of formamide at $55^{\circ} \mathrm{C}$ for $24 \mathrm{~h}$. The absorbance of the eluted dye was then analyzed by a plate reader (Tecan, Grödig, Austria) at $620 \mathrm{~nm}$ along with standards for calculation of dye concentration.

Mouse model of chronic skin inflammation

For oxazolone-induced psoriasis-like skin inflammation in the ear skin [22], K14-VEGF-A ${ }^{+/-}$transgenic mice ( $n=5$, females, 10 weeks age) were anesthetized by i.p. injection of $0.2 \mathrm{mg} / \mathrm{kg}$ medetomidine and $80 \mathrm{mg} / \mathrm{kg}$ ketamine, and $2 \%$ oxazolone (4-ethoxymethylene-2 phenyl-2oxazoline-5-one; Sigma-Aldrich) in acetone/olive oil (4:1 $v / v)$ was applied topically to the shaved abdomen $(50 \mu \mathrm{L})$ and to each paw $(5 \mu \mathrm{L})$. Five days after sensitization (day 0 ), the right ear was challenged by topical application of $10 \mu \mathrm{L}$ oxazolone (1\%) and the left ear remained untreated as a control. Mice were imaged with the IVIS before sensitization and at days 2, 9, 16, 23, and 30 after challenge. Ear thickness was measured on the same days as imaging using calipers. One mouse had obvious signs of inflammation in the control ear (increased swelling and redness) and therefore was excluded from the analyses.

\section{T1 model of breast cancer}

4T1 mammary carcinoma cells (ATCC) were maintained in DMEM-Glutamax (Invitrogen), supplemented with $10 \%$ FCS (Invitrogen). Cells were retrovirally transduced with MSCV-IRES-Venus (kind gift from Dr. Johannes Zuber, IMP, Vienna, Austria). Retroviral supernatants were produced by transiently transfecting the HEK-293T packaging cell line by standard calcium phosphate transfection techniques. Harvested supernatants were filtered through a $0.45-\mu \mathrm{m}$ filter and stored at $-80{ }^{\circ} \mathrm{C}$. $4 \mathrm{~T} 1$ cells were cultured in the presence of retrovirus-containing supernatants and polybrene $(4 \mu \mathrm{g} / \mathrm{mL})$ for $12 \mathrm{~h}$ each for a total of 6 transfection cycles. Following the last incubation, cells were cultured for at least $72 \mathrm{~h}$. FACS sorting was employed to obtain a stable population of 4T1-MSCV Venus cells with a high level of fluorescence. 4T1-MSCVVenus cells $\left(5 \times 10^{4}\right)$ were injected subcutaneously into the rear right flank of 8-week old female Balb/c mice (Janvier, Le Genest Saint Isle, France). Mice were imaged at 14 days (with IVIS) after tumor inoculation at which time tumors were approximately $8-10 \mathrm{~mm}$ in diameter.

\section{Statistics}

Statistical analysis was performed using Prism version 4.03 (GraphPad Software, Inc.). Data are shown as mean \pm SD and were analyzed with a two-tailed unpaired Student's $t$ test. When more than two groups were compared, ANOVA was applied and the individual groups were compared using a Tukey-HSD post hoc test.

\section{Results}

Development of NIR vascular leakage tracers

We first designed NIR dye-conjugates to identify a vascular tracer for imaging with the desired features of stable vascular signals at early time points after injection and a suitable clearance profile. The molecular weights of the polymeric backbone of the tracers ( 20 or $40 \mathrm{kDa}$, Table 1) were chosen in order to avoid a prolonged circulation lifetime that would have hampered longitudinal studies. The tracers (P20-D800 or P40-D800) were prepared by coupling the PEG to the NIR dye IRDye ${ }^{\circledR} 800 \mathrm{CW}$ (D800).

We next evaluated the PK profiles in serum and biodistribution of the two conjugates (Table 1, Supplementary Figs. 1 and 2). We sampled blood at select time points after injection of the tracers (Supplementary Fig. 2) and determined terminal serum half-lives of $2 \mathrm{~h} 25 \mathrm{~min}$ for P20D800 and 12 h 35 min for P40-D800 (Table 1). The relatively slow elimination, as expected for a macromolecule, resulted in almost constant tracer concentration in blood for the first $15 \mathrm{~min}$ after the injection. The apparent volume of distribution $\left(\mathrm{V}_{\mathrm{d}}\right)$ of both tracers was found to be similar to the total serum volume of mice, indicating that the NIR dye-conjugates remained initially in the bloodstream and did not distribute rapidly to the tissues. Both tracers were excreted in intact form through renal filtration (Supplementary Fig. 2). Lower signals were seen in all evaluated tissues in the P20-D800 injected versus the P40-D800 animals at one week post injection, confirming the much slower clearance of the latter (Supplementary Fig. 2d).

Table 1 Pharmacokinetic parameters of P20-D800 and P40-D800 after intravenous administration

\begin{tabular}{|c|c|c|c|c|c|c|c|}
\hline Sample & Calculated RH (nm) & $\mathrm{M}_{\mathrm{n}} / \mathrm{NMR}(\mathrm{kDa})$ & $\mathrm{K}_{\mathrm{el}}\left(\mathrm{h}^{-1}\right)$ & $\mathrm{t}_{1 / 2}(\mathrm{~h})$ & $\operatorname{AUC}_{0-\infty}(\mu \mathrm{mol} \times \mathrm{h} / \mathrm{mL})$ & $\mathrm{CL}(\mathrm{mL} / \mathrm{h})$ & $\mathrm{V}_{\mathrm{d}}(\mathrm{mL})$ \\
\hline P20-D800 & 4.2 & 21.5 & $0.30 \pm 0.06$ & $2.43 \pm 0.73$ & $2.71 \pm 0.34$ & $0.54 \pm 0.10$ & $1.81 \pm 0.15$ \\
\hline P40-D800 & 5.8 & 37.9 & $0.058 \pm 0.019$ & $12.6 \pm 4.3$ & $14.4 \pm 3.0$ & $0.09 \pm 0.02$ & $1.62 \pm 0.21$ \\
\hline
\end{tabular}

Administered dose (expressed as D800 content): $0.05 \mathrm{nmol} / \mathrm{g}$ body weight. Data are expressed as mean $\pm \mathrm{SD}(n=3)$ 
We then collected a dynamic series of NIR images after intravenous administration in normal mice that allowed real-time assessment of signal intensities of the fluorescent tracers compared to the established tracer ICG. ICG showed immediate saphenous vein vessel enhancement followed by rapid decreases in blood vessel intensity, while P20-D800 and P40-D800 dye conjugates reached a quasiequilibrium in the bloodstream rapidly with almost no change in signal during the duration of imaging (Fig. 1a). In ear tissue, ICG signal rapidly decreased after an initial enhancement, while P20-D800 showed a slowly increasing signal indicating that the conjugate leaked out of normal skin blood vessels but at a very low rate (Fig. 1b). P40D800 showed stable signal similar to that seen at the saphenous vein, suggesting that this tracer remains predominantly intravascular during the period of imaging.

P20-D800 cleared faster from the ear tissue than P40D800 (Fig. 1c, d), allowing longitudinal imaging with low background at earlier time points. At $72 \mathrm{~h}$ after injection, mice injected with P20-D800 had approximately $20 \%$ of the signal intensity in the ear that was seen at $3 \mathrm{~h}$, while P40-D800 injected animals retained around $80 \%$ of the signal. Based on the stability of fluorescence signals at early time points and the clearance profile, we mainly focused our subsequent experiments on P20-D800.
Quantification of tissue vascularity and leakage

We assumed a two-compartment model for tissue to develop quantifications of vascular leakage, in which the first compartment was defined as the intravascular compartment (blood) of the tissue and the second was defined as the extravascular compartment (comprised of interstitium and lymphatic vessels) (Fig. 2a). Based on the revised Starlings forces and the size of the fluorescent tracers, we assumed that there was one-way movement of the tracers from the intravascular to the extravascular compartment and that return of the tracers to the blood via the venous system was negligible [2]. We also assumed that clearance out of the tissue of the leaked tracers by the lymphatic vessels would be negligible in the initial timepoints. By performing simultaneous dynamic imaging on both the saphenous vein region (assumed as $100 \%$ intravascular) and the ears (Fig. 2b), we were then able to derive quantifications based on normalization of the tissue fluorescence signal to the blood vascular signal. This allowed for an estimation of the blood volume fraction of the tissue shortly after the injection, and with dynamic imaging, an assessment of the increase in extravascular signal over time.

The quantifications were initially developed in normal mice after tail vein injection of P20-D800. By plotting of
Fig. 1 Development of a suitable vascular leakage tracer. a Comparison of the dynamics after tail vein injection of ICG $(n=10), \mathrm{P} 20-\mathrm{D} 800(n=10)$, or P40-D800 $(n=6)$ into normal mice. Normalized saphenous vein signals plotted over time after i.v. injection. b Normalized ear signals after i.v. injection. c Representative images of the ears after i.v. injection to show the clearance of signal over time between P20-D800 and P40-D800.

d Quantification of the clearance of signal (mean $\pm \mathrm{SD}$ ) in the ears for $n=3$ mice between P20-D800 and P40-D800. Data is normalized to the ear signal intensity at $3 \mathrm{~h}$. $* p<0.05$, $* * p<0.01, * * * p<0.001$
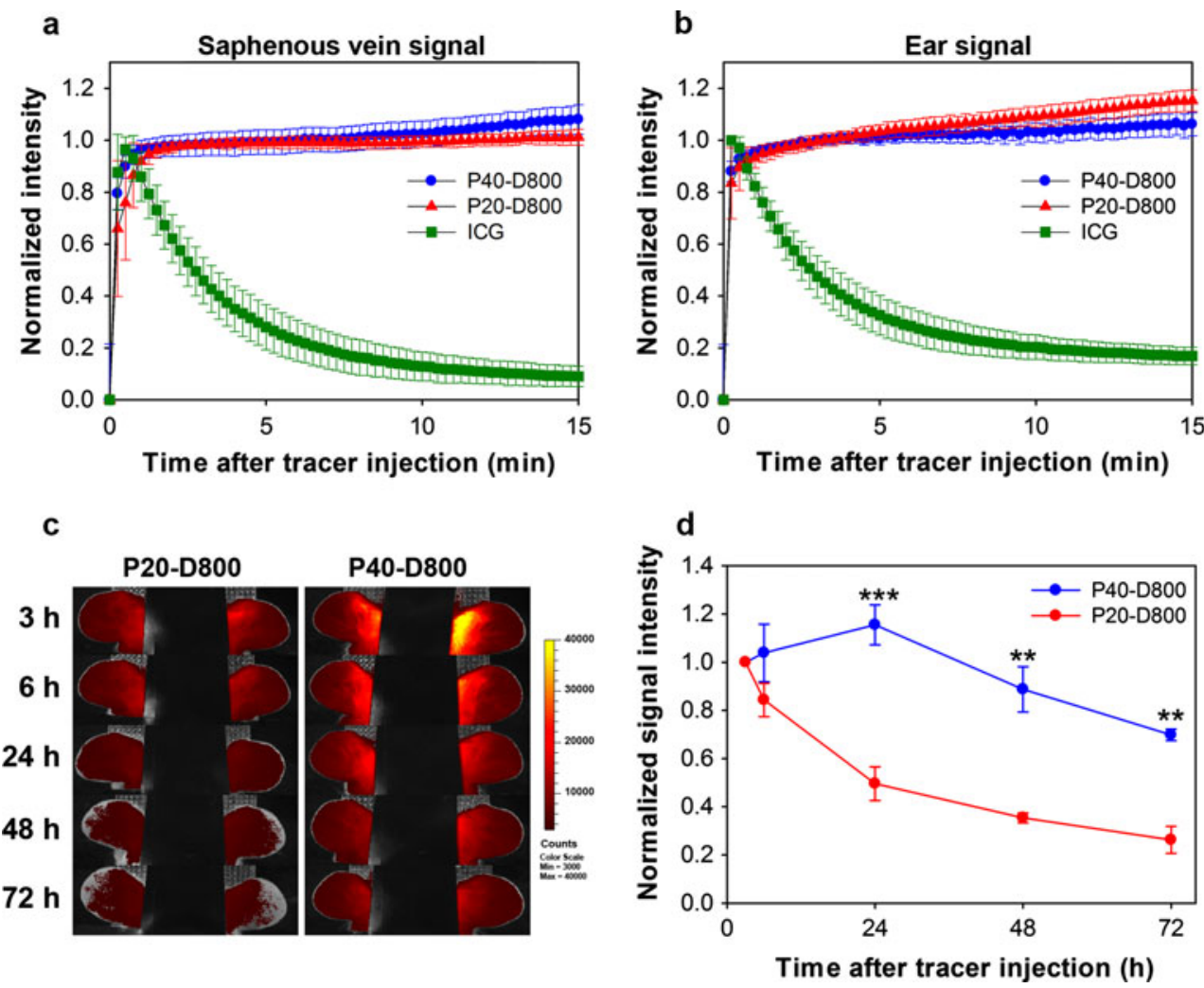
Fig. 2 Quantifications of blood volume fraction, tissue leakage rate and vascular leakage rate in normal mice. a Twocompartment model for development of quantifications of vascular leakage using NIR tracers. b Sequence of images demonstrating the animal positioning and dynamics of P20-D800 signal in the saphenous vein and ears in a normal mouse for measurement of vascular leakage. c Signal intensity plots for regions of interest over the saphenous vein and ear exhibit stable fluorescence intensities after injection. d Plot demonstrating measurements of tissue leakage rate (slope of linear fit equation) and blood volume fraction (indicated by arrow). Units are presented as ratio of ear signal enhancement (ESE) to saphenous vein signal enhancement (SVSE). e Normalized plot showing measurement of vascular leakage rate (slope of linear fit equation). Units are presented as ratio of normalized ear signal enhancement (NESE) to normalized saphenous vein signal enhancement (NSVSE) a

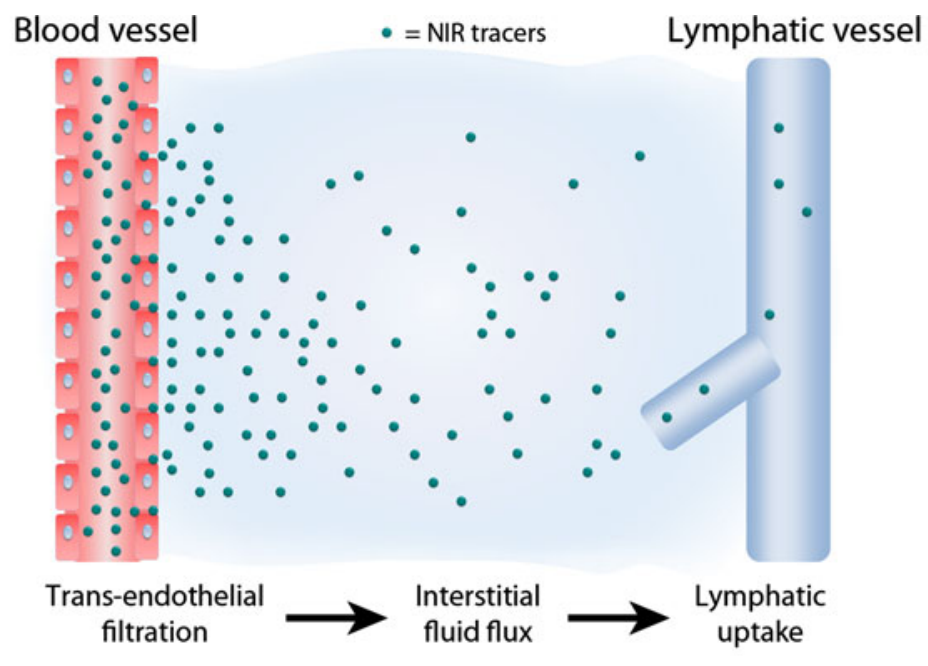

\section{Intravascular (Blood) \\ Extravascular (Interstitium + Lymphatics)}

\section{b}

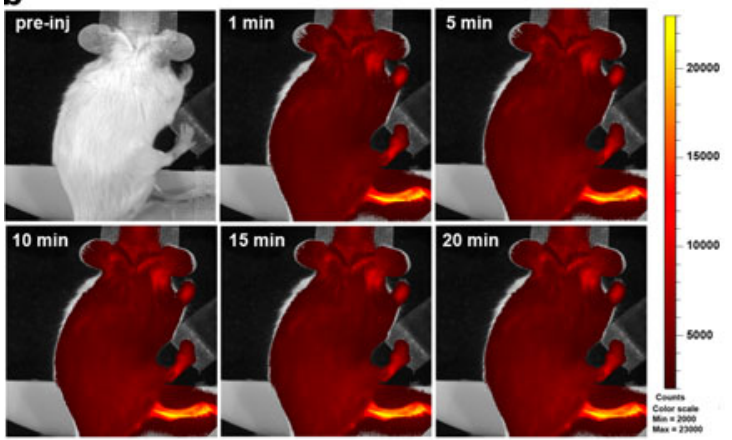

C

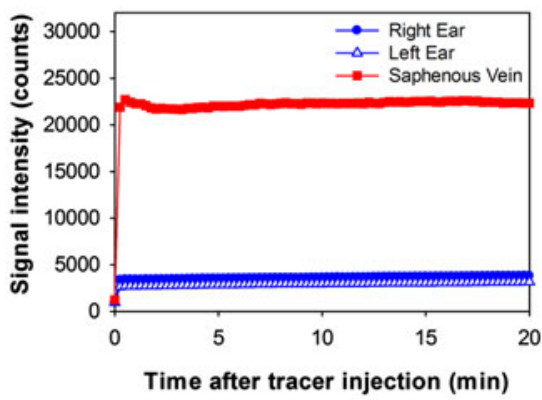

d

Tissue leakage rate (TLR)

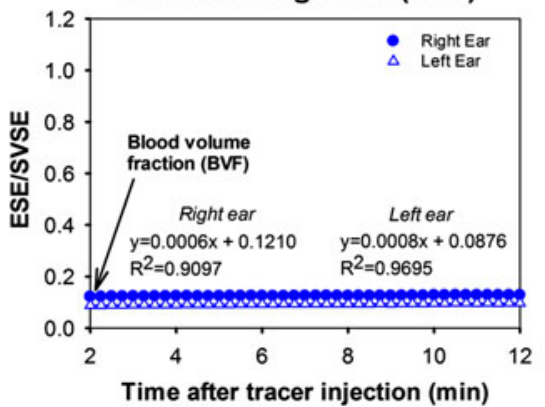

fluorescent signal intensities over time from regions of interest drawn on the ears and saphenous vein (Supplementary Fig. 3), we found that there were no effective changes in signal from the time quasi-equilibrium was reached in the bloodstream at $\mathrm{t}=1 \mathrm{~min}$ until $\mathrm{t}=20 \mathrm{~min}$ (Fig. 2c). However, when the ear signal enhancement (ESE) values were normalized to the saphenous vein signal enhancement (SVSE) values, there were positive slopes over time indicating slow leakage of P20-D800 into tissue (Fig. 2d). From this plot we derived two quantifications based on the assumptions of the two-compartment model.
The first, calculated at the time point that blood signal equilibrium was reached after the i.v. injection, was termed blood volume fraction (BVF) and represents an estimate of the intravascular compartment (perfused blood vessels) in the tissue relative to the region of assumed $100 \%$ vascularity, the saphenous vein. Normal mouse ears typically displayed blood volume fraction values of approximately 0.1 (i.e. $10 \%$ vascularity). The second value was defined as the slope of the ESE/SVSE values over time and was termed tissue leakage rate (TLR, expressed in $\min ^{-1}$ ). This value represents the increase in signal in the extravascular 
compartment over time. Normal mouse ears exhibited a very low TLR of P20-D800 tracer ( $\sim 0.1 \%$ of the ESE/ SVSE per min). Since tissue leakage rates are dependent on the surface area of the blood vessels available for filtration, it was necessary to adjust TLR by normalization to BVF (Fig. 2e), to calculate the vascular leakage rate (VLR, expressed in $\min ^{-1}$ ). It is important to note that the calculated leakage rates will be dependent on not only vascular permeability but also on the rates of interstitial fluid flux and lymphatic uptake.

We next compared the BVF, TLR, and VLR values in normal mice that were injected with either P20-D800 or P40-D800 (Supplementary Fig. 4a). We found that mice injected with the smaller tracer P20-D800 had significantly higher TLR $(p<0.001)$ and VLR $(p<0.01)$ values compared to mice injected with P40-D800, with no significant difference in BVF. Using a stereomicroscope adapted for far-red wavelengths for higher resolution dynamic visualization of the leakage in the ear, we validated that P20 dye conjugates are leaking into normal skin tissue, while P40 dye conjugates appear to stay intravascular during the 30 min after injection (Supplementary Fig. 4, Supplementary Movies 1 and 2). Thus, these findings reveal that P20D800 showed steady-state leakage out of blood vessels into normal skin tissue.

Quantification of vascularity and leakage in transgenic mice with overexpression of VEGF-A or VEGF-C in the skin

We next compared wildtype mice with transgenic mice that express murine VEGF-A164 specifically in the skin under control of the keratin-14 promoter. K14-VEGF-A homozygous mice (K14-VEGF-A ${ }^{+/+}$) have increased blood vascular density in the skin with enhanced vascular leakage as shown with the Miles assay and development of skin inflammation resembling psoriasis [18, 19]. K14-VEGF-A hemizygous mice (K14-VEGF-A ${ }^{+/-}$) have less expression of VEGF-A and show increased skin vascularity over normal mice but no spontaneous development of inflammation [22, 23]. We also analyzed K14-VEGF-C transgenic mice that have been reported to have an expanded lymphatic vessel network with no changes in blood vascular density [17].

After injection of P20-D800, the increased vascularity in the K14-VEGF-A ${ }^{+/+}$mice was clearly seen compared to the wildtype at the $1 \mathrm{~min}$ time point. A slight increase in vascularity could be visualized in the $\mathrm{K} 14-\mathrm{VEGF}_{-} \mathrm{A}^{+/-}$ mice. The increased tracer leakage in the K14-VEGF-A ${ }^{+/+}$ mice over the other genotype mice was apparent by the increased blurring and brightness seen at later time points (Fig. 3a). TLR graphs (Fig. 3b) show increases in slopes in the K14-VEGF-A ${ }^{+/-}$and K14-VEGF-A ${ }^{+/+}$mice compared to wildtype (Fig. 2c) and K14-VEGF-C mice, reflecting the increased leakage of $\mathrm{P} 20-\mathrm{D} 800$ in these mice.

With quantifications (Fig. 3c), K14-VEGFA ${ }^{+/-}$mice demonstrated a 1.8-fold increase in BVF over wildtype controls $\left(p<0.01\right.$ ), whereas K14-VEGF-A ${ }^{+/+}$mice had a more variable 6.5-fold increase $(p<0.001)$. K14-VEGF-C mice showed no difference in BVF compared to wildtype. TLR was sixfold increased in K14-VEGF-A ${ }^{+/-}$mice compared to wildtype mice $(p<0.001)$. In K14-VEGF$\mathrm{A}^{+/+}$mice, TLR values were significantly higher than both wildtype (31-fold, $p<0.001$ ) or K14-VEGF-A ${ }^{+/-}$mice (5.2-fold, $p<0.001)$. The high variability seems to be inherent in the K14-VEGF-A ${ }^{+/+}$model, as examination of the ears of the mice showed obvious inflammation with different degrees of vascularity and redness, even between ears of the same animal (Supplementary Fig. 5).

After normalization for the differences in BVF, we found a highly significant 3.3-fold increase of VLR $(p<0.001)$ in $\mathrm{K} 14-\mathrm{VEGF}-\mathrm{A}^{+/-}$versus wildtype mice (Fig. 3c). K14-VEGF-A ${ }^{+/+}$mice also displayed significant increases in VLR over wildtype (4.7-fold, $p<0.001$ ) and

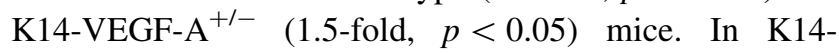
VEGF-C transgenic mice, we found no significant differences in TLR and VLR compared to wildtype controls. These results confirmed that the developed imaging method is able to adequately detect changes in vascularity and leakage in different genetic mouse models.

Reliable quantification of the changes in vascularity and leakage during the course of chronic inflammation

We next measured the inflammation-associated blood vascular changes over time in K14-VEGF-A ${ }^{+/-}$mice in response to a contact hypersensitivity reaction induced with oxazolone [24]. In this model of psoriasis-like inflammation, a strong increase in ear swelling is seen within hours after antigen challenge. Peak swelling is reached around Day 2 after challenge and slowly diminishes over time, allowing serial imaging of the vascular response (Fig. 4a). We challenged the right ear with oxazolone and the left ear remained untreated as a control. Imaging was performed before challenge and every 7 days starting at Day 2.

A dramatic increase in tissue leakage in the oxazolonechallenged right ear was seen $20 \mathrm{~min}$ after intravenous injection of P20-D800 at Day 2 of inflammation (Fig. 4b). Repeated imaging of the same mouse showed diminishing leakage on Days 16 and 30 after challenge. In contrast, the unchallenged left ear showed no obvious differences in leakage at the different time points (Fig. 4b). Quantifications (Fig. 4c) demonstrated highly significant increases in the challenged ear compared to the unchallenged ear in BVF (2.4-fold, $p<0.001)$ at Day 2. BVF peaked at Day 9 

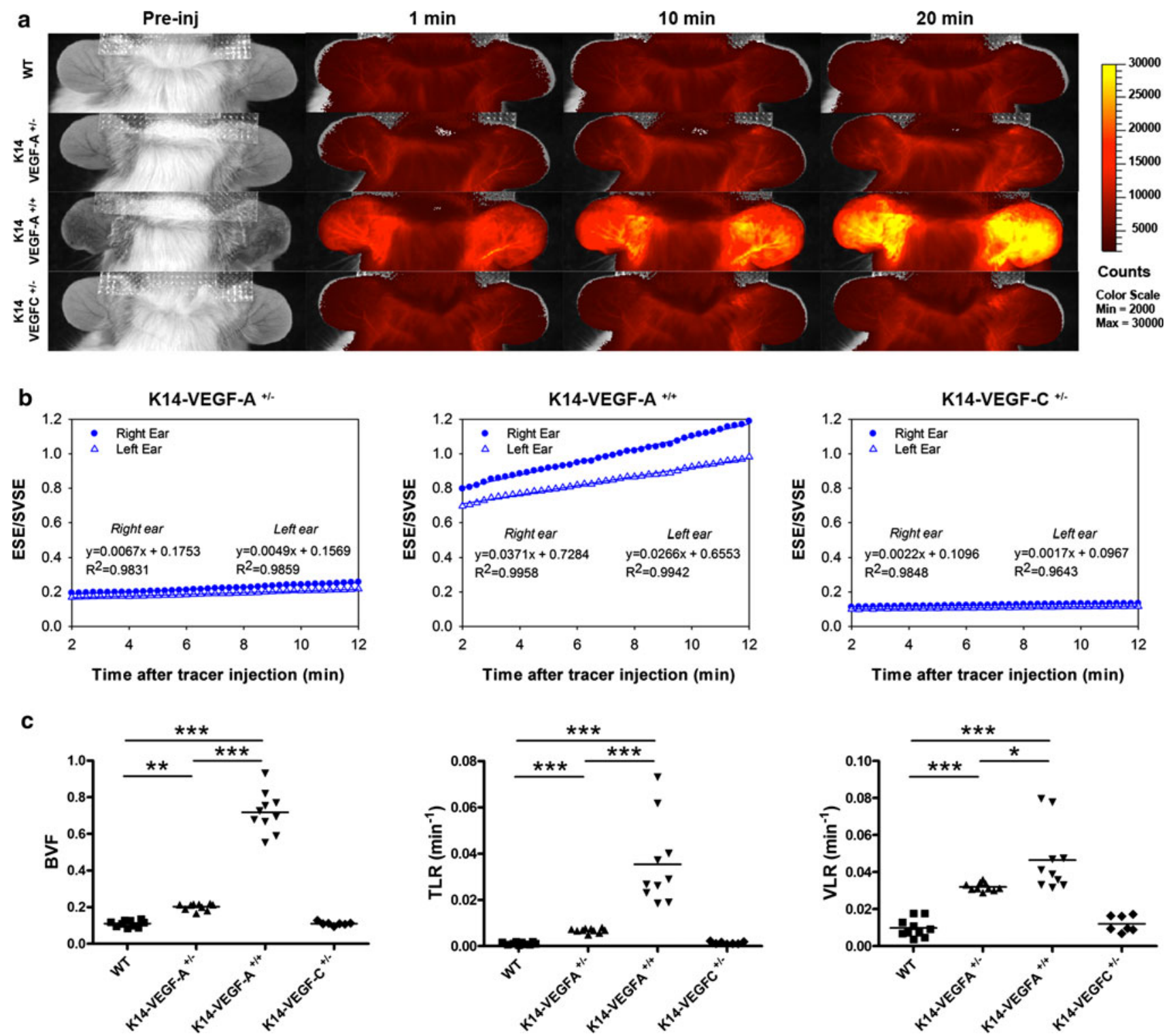

Fig. 3 Vascularity and leakage in transgenic mice with overexpression of VEGF-A and VEGF-C in the skin. a Representative images of ears from wildtype, K14-VEGF-A ${ }^{+/-}$, K14-VEGF-A ${ }^{+/}$and K14VEGF-C $^{+/-}$transgenic mice at pre-injection, 1, 10, and $20 \mathrm{~min}$ after injection of P20-D800 tracer. b Sample plots of tissue leakage rates

(2.8-fold, $p<0.01)$ and then declined slowly over time with no significant difference $(p=0.07)$ to unchallenged ears at Day 30 after challenge. We found that TLR peaked at Day 2 in challenged ears (5.2-fold over control, $p<0.001)$ and then decreased steadily over time. At Day 30, TLR was still significantly higher than in unchallenged ears $(1.8$-fold, $p<0.05)$. VLR was significantly higher at Day 2 (2.3-fold over control, $p<0.001)$ and then showed no significant difference compared to control values as early as Day 9 after challenge. Ear thickness showed a closer correlation with TLR than with BVF or VLR over time (Supplementary Fig. 6). These results indicate that the from K14-VEGF-A ${ }^{+/}$(left), K14-VEGF-A ${ }^{+/+}$(middle), and K14VEGF-C $^{+/-}$(right). c Distribution plots showing quantifications of BVF, TLR, and VLR for groups $(n=5)$ of transgenic mice. $* p<0.05, * * p<0.01$, *** $p<0.001$

in vivo imaging with P20-D800 can reproducibly track vascularity and leakage changes in longitudinal studies of chronic inflammation.

K14-VEGF-C mice demonstrate increased leakage rates after VEGF-A injection

We next investigated whether the developed imaging method was sensitive enough to quantify changes in vascular leakage in response to injections of VEGF-A. Vascular leakage has been reported to be acutely induced within a few minutes of injection of VEGF-A due to 
Fig. 4 Validation of reproducibility and longitudinal imaging in chronic skin inflammation model. a Timeline of induction and imaging points of the contact hypersensitivity model in K14-VEGF-A ${ }^{+/}$ mice. $\mathbf{b}$ Sample images of one mouse taken at $20 \mathrm{~min}$ after injection of P20-D800 tracer at Day 2 (left), Day 16 (middle), and Day 30 (right). Right ear was challenged with oxazolone while the left remained unchallenged. c Quantifications (mean $\pm \mathrm{SD}$ ) over time in same cohort of mice $(n=5)$ for $\mathrm{BVF}$ (upper left), TLR (upper right), VLR (bottom left), and ear thickness (bottom right). $* p<0.05, * * p<0.01$, $* * * p<0.001$ versus control ears a Contact hypersensitivity model with K14-VEGFA ${ }^{+/-}$mice:
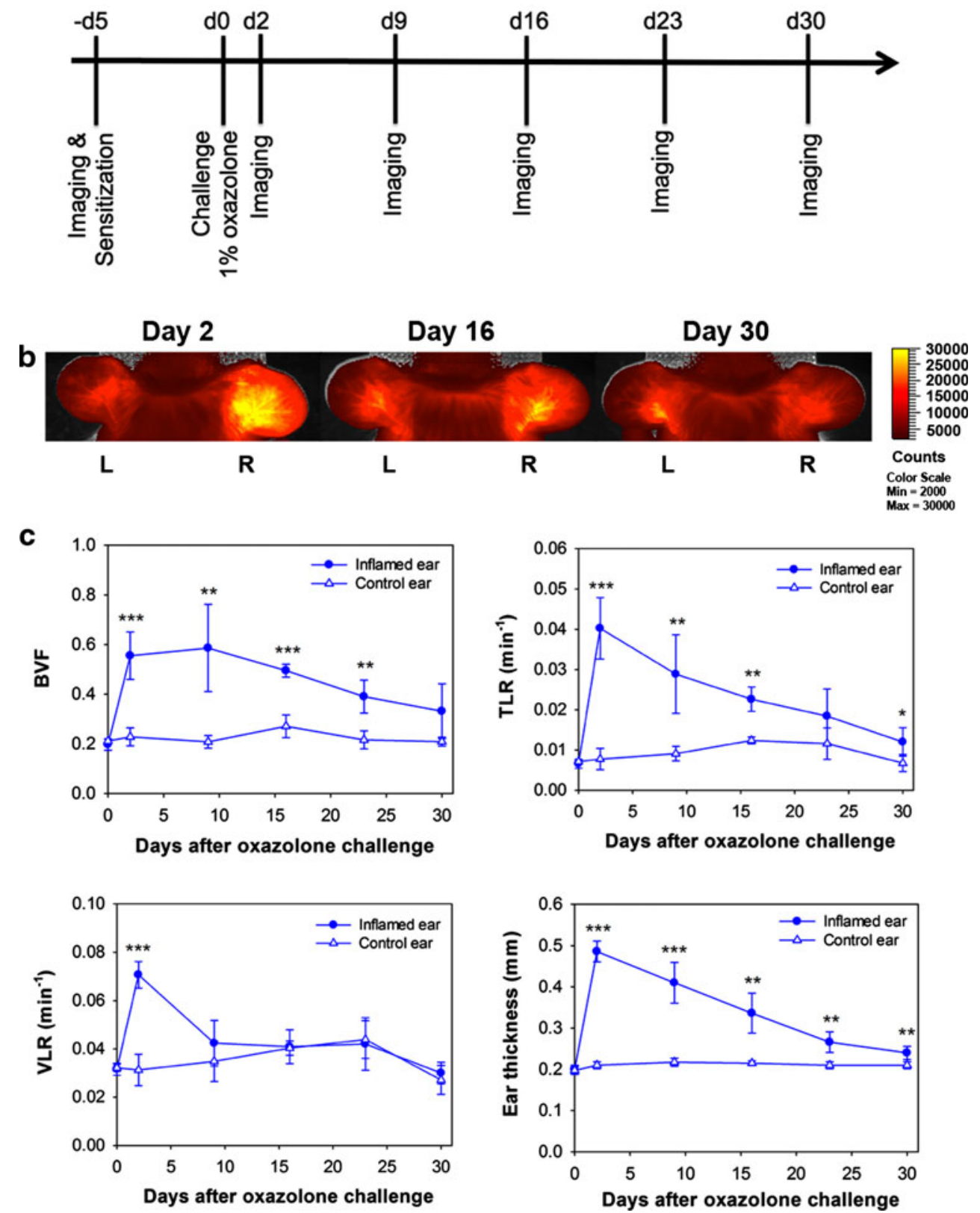

vasodilation and increases in blood vessel permeability [7]. This is followed by rapid internalization and deactivation of the growth factor by its receptor VEGFR2 and attenuation of the leakage effect [7, 25].

We first analyzed vascular leakage after intradermal injections of $30 \mathrm{ng}$ human VEGF-A165 or saline control into wildtype and K14-VEGF-C mouse ears (Fig. 5a). A traditional Miles assay in wildtype mice with this protocol resulted in a significant 3.2-fold increase $(p=0.05)$ in Evans Blue dye concentration in the VEGF-A injected ear over the control ear at $30 \mathrm{~min}$ after injection (Supplementary
Fig. 7). For imaging of this response, we initiated our imaging sequence with tail vein injections of P20-D800 tracers approximately $3 \mathrm{~min}$ after the ear injections. In wildtype mice, a minor increase (1.3-fold, $p=0.16)$ was found in BVF in the VEGF-A injected ears over the saline injected ears. In K14-VEGF-C mice, the increase in BVF was significant $(1.7$-fold, $p<0.01)$. Significant increases were found in both TLR (4.7-fold, $p<0.05)$ and VLR (3.4fold, $p<0.001)$ in the VEGF-A injected ears in wildtype animals. In K14-VEGF-C mice, we also found significant increases in both TLR (8.2-fold, $p<0.001)$ and VLR 

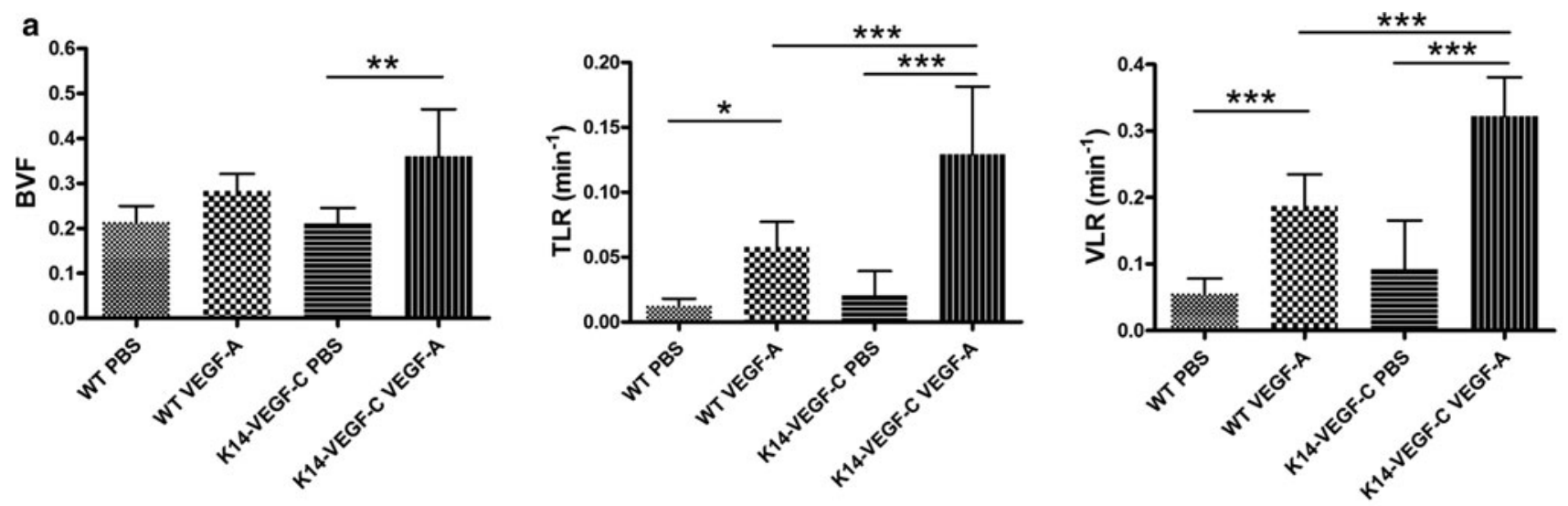

b

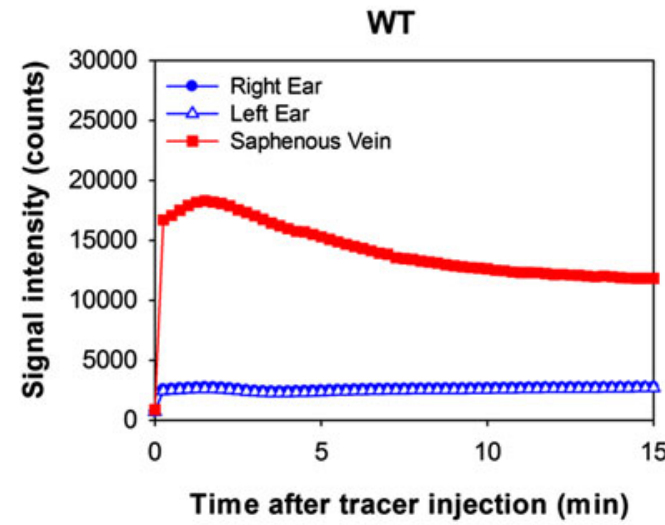

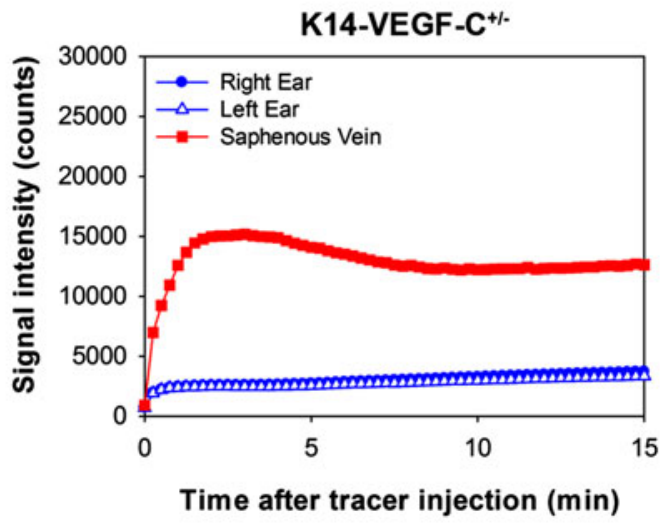

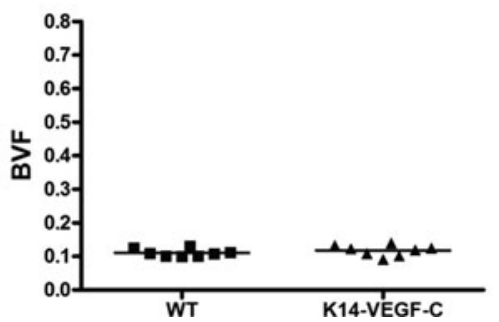

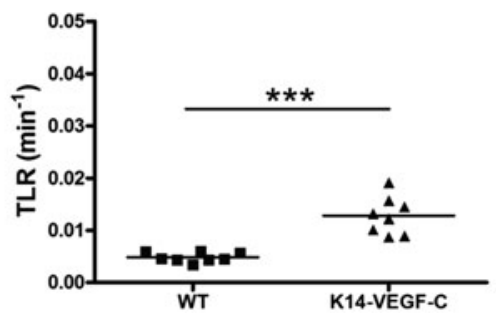

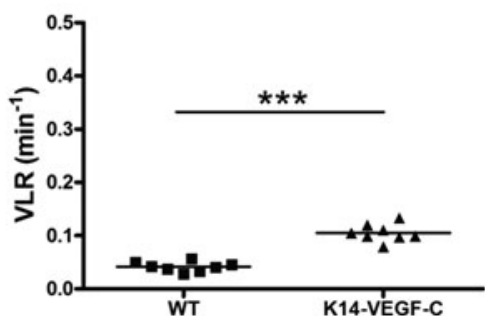

Fig. 5 Acute permeability measurements after injections of human VEGF-A165. a Quantifications (mean \pm SD) demonstrating differences between wildtype and K14-VEGF-C ${ }^{+-}(n=5)$ mice after injections of 30 ng VEGF-A or PBS into the ear skin. b Sample signal intensity plots after tail vein injections of $1 \mu \mathrm{g}$ VEGF-A and P20-
D800 tracer in wildtype (left) and K14-VEGF-C ${ }^{+/-}$(right). c Quantifications demonstrating differences in BVF (left), TLR (middle), and VLR (right) between wildtype and K14-VEGF-C ${ }^{+/-}$mice after tail vein injections of $1 \mu \mathrm{g}$ VEGF-A. * $p<0.05$, ** $p<0.01$, $* * * p<0.001$ (3.6-fold, $p<0.001$ ) in VEGF-A injected ears. K14-VEGF$\mathrm{C}$ mice exhibited significantly increased TLR values (2.2fold, $p<0.001$ ) over wildtype animals in response to VEGF-A, indicating that leakage is enhanced in these animals. We found that in K14-VEGF-C mice, the leakage was occurring so rapidly in response to VEGF-A that it was impossible to quantify accurately the BVF values, as leakage had begun prior to the time point when equilibrium was reached in the bloodstream. Despite these increased BVF values, which may also reflect to some extent vascular dilation, we were able to detect a significant higher VLR (1.7-fold, $p<0.001$ ) in K14-VEGF-C animals over wildtype after VEGF-A injection.

Since intradermal injections may cause vascular damage and significant leakage occurred even in saline injected tissue, we next performed imaging after tail vein injection of $1 \mu \mathrm{g}$ human VEGF-A165 in conjunction with P20-D800 dye. As seen in Fig. 5b, the saphenous vein signal in both wildtype and K14-VEGF-C mice showed vascular dilation in the initial 2 to $3 \mathrm{~min}$, followed by a steady decrease in fluorescence, likely due to systemic leakage effects. During the time directly after maximum enhancement was 
reached, leakage rates in the ear skin were quantified in wildtype and K14-VEGF-C mice (Fig. 5c). After intravenous administration of VEGF-A, leakage occurred at a slower rate than after skin injection and BVF values could therefore be calculated with more accuracy. With this protocol, we found no significant difference in BVF ( $p=0.34$ ) between wildtype and K14-VEGF-C mice. However, we again found significant increases in TLR (2.7-fold, $p<0.001)$ and VLR (2.5-fold, $p<0.001)$ in K14-VEGF-C over wildtype mice. Using high-resolution dynamic stereomicroscopy we confirmed these results. After ears of wildtype and K14-VEGF-C mice were positioned under the microscope, the mice were injected with $1 \mu \mathrm{g}$ of VEGF-A and P20-D680 into the tail vein. As shown in Supplementary Movies 3 and 4, the K14-VEGF$\mathrm{C}$ mouse ear has increased spreading of signal around the blood vessels over time, indicating faster leakage compared to the wildtype mouse.
Expanded dermal lymphatic network and rapid lymphatic clearance after acute permeability reaction in K14-VEGF-C mice

The unexpected finding of increased vascular leakage rates during an acute permeability reaction seen in the K14VEGF-C mice led us to investigate these mice further. To visualize the lymphatic network of the ear, we performed fluorescence lymphangiography with a stereomicroscope after an injection of P40-D680 tracer into the tip of the ear (Fig. 6a). Immediately after the injection, the tracer filled an expanded dermal network of lymphatic vessels with no uptake into blood vessels. The lymphatic network appeared to fill most of the available space with the exception of the hair follicles, as described previously [26]. The tracer exhibited rapid lateral spreading throughout this dermal network and eventual absorption into the collecting vessels of the ear. By comparison, the wildtype mice demonstrated
Fig. 6 Visualization of the lymphatic vasculature in the ears and clearance of tracer after acute leakage.

a Lymphangiography after intradermal injection of P40D680 into the ears of wildtype (left) and K14-VEGF-C (right) mice. Inset in right panel shows higher magnification of expanded lymphatic capillaries in the $\mathrm{K} 14-\mathrm{VEGF}-\mathrm{C}^{+/-}$ear. b Representative lymphatic clearance of P20-D800 (normalized to ear signal enhancement at 40 min after i.v. injection) from VEGF-A injected ears in wildtype (left) and $\mathrm{K} 14-\mathrm{VEGF}-\mathrm{C}^{+/-}$(right) mice. $\mathrm{R}^{2}$ values show close fit to a model of one-phase exponential decay.

c Quantifications of half-life and $\mathrm{K}$ rate values decay curves as measures of lymphatic clearance in wildtype $(n=5)$ and K14-VEGF-C ${ }^{+/-}(n=4)$ mice. $* p<0.05$, ** $p<0.01$, *** $p<0.001$
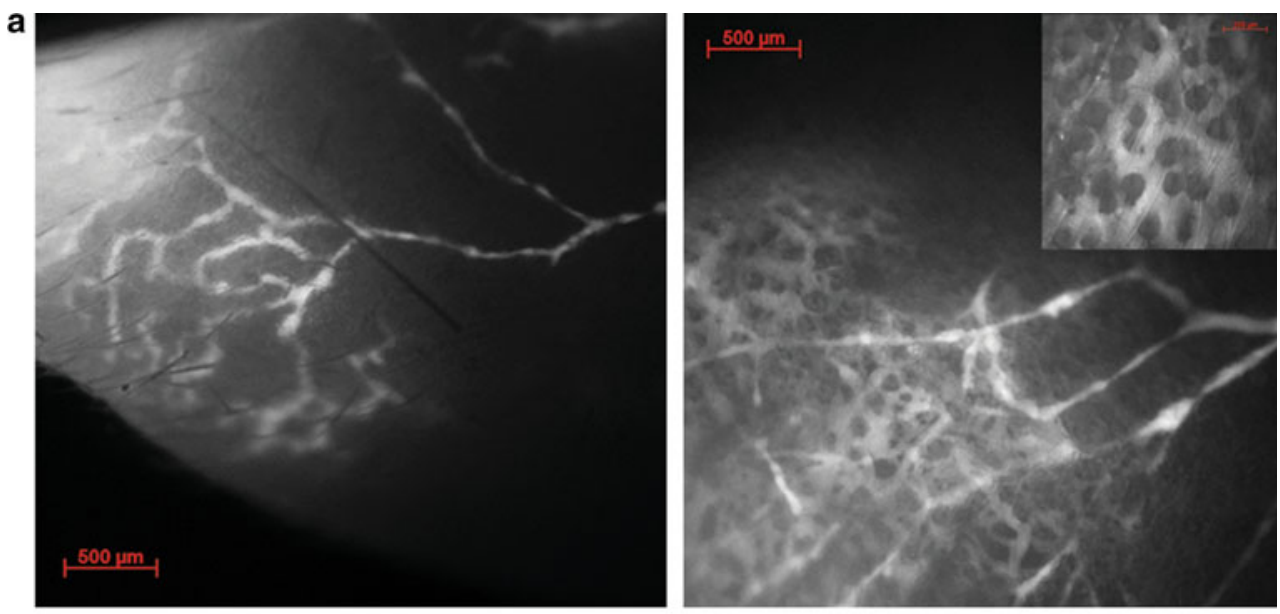

b

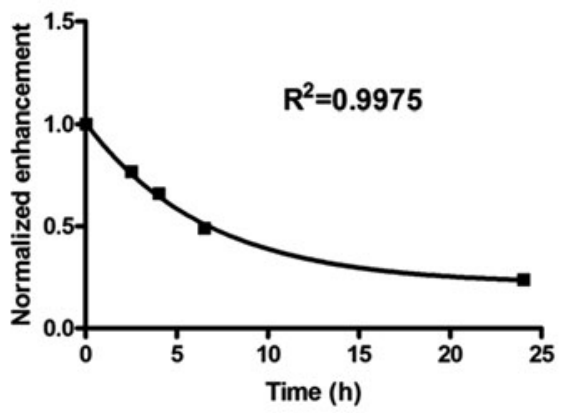

C

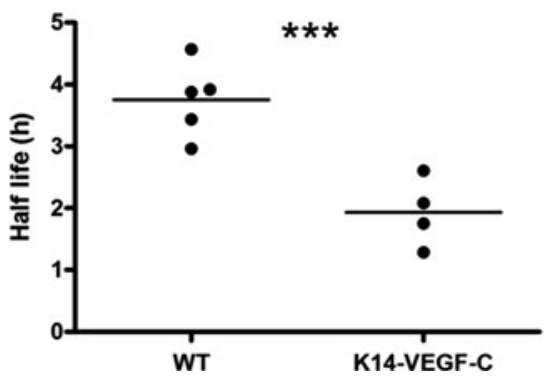

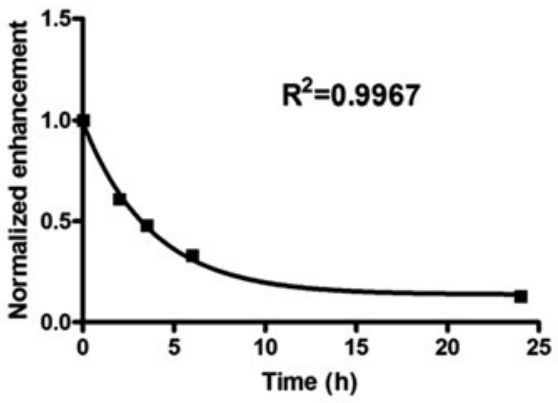

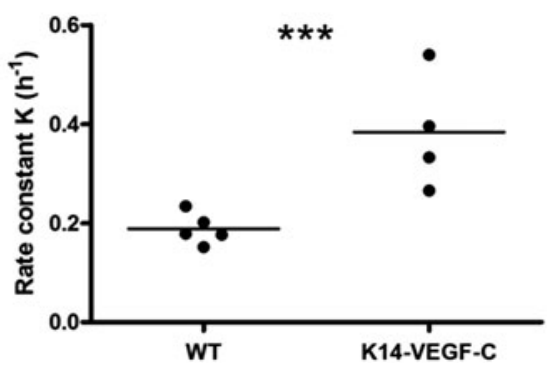



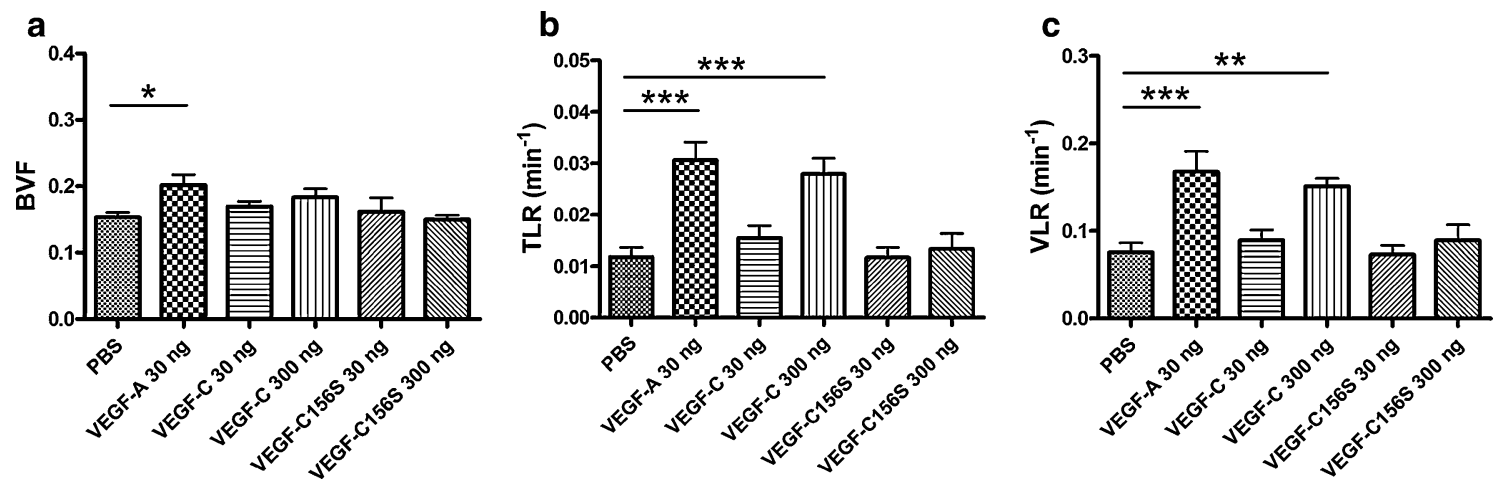

Fig. 7 Acute permeability measurements after ear injections of native and mutant forms of fully processed human VEGF-C $(n=6)$. a Quantifications (mean $\pm \mathrm{SD}$ ) of BVF. b TLR. c VLR. $* p<0.05$, ** $p<0.01$, *** $p<0.001$

a less dense network of lymphatic capillaries with no lateral spreading of the tracer.

The expanded network of lymphatic vessels in K14VEGF-C mice has been suggested to be dysfunctional and associated with retrogradal flow [17, 26]. However, we recently observed increased lymphatic clearance in these mice over wildtype controls at $16 \mathrm{~h}$ after intradermal Evans Blue dye injection [27]. To further investigate this issue, we developed a protocol in which the decay of signal in the ear is monitored after tracer leakage from blood vessels is induced by an acute permeability reaction to VEGF-A. The permeability effect is short-lived after injection of VEGFA (Supplementary Fig. 8) and therefore vascular leakage of the tracer at later time points is considered to be negligible $[4,25]$. Therefore, this approach may quantify lymphatic clearance in a more relevant setting compared to clearance directly after tracer injections into ear skin, which likely cause supraphysiological interstitial pressures and overloading of lymphatic vessels [28, 29]. As shown in Fig. 6b, a representative K14-VEGF-C mouse had enhanced tissue clearance of the tracer signal compared to wildtype. These data very closely fit $\left(\mathrm{R}^{2}>0.99\right)$ to a one-phase exponential decay model in which the clearance of signal can be expressed by rate constant $\mathrm{K}$ or by half-life quantifications (Fig. 6c). The average half-life of clearance in K14-VEGF$\mathrm{C}$ mice was significantly shorter $(p<0.001)$ than in wildtype mice with $\mathrm{K}$ rates significantly higher $(p<0.001)$, indicating that the lymphatic clearance of the extravasated tracer is accelerated in these mice.

The mature form of VEGF-C induces vascular leakage via VEGFR2

We next investigated whether human VEGF-C can have acute effects on vascular leakage by injecting both non-mutated and mutated forms of fully processed VEGF-C into the ear and tail veins of wildtype mice. The non-mutated fully processed form of VEGF-C has been shown to activate VEGFR-3 and
VEGFR-2 while the mutant form VEGF-C156S is only able to activate VEGFR-3 [30]. We first tested these proteins at the same dose (30 ng of protein injected into the ear skin) that was used to induce leakage with VEGF-A. As shown in Fig. 7, we found increased TLR and VLR values with this dose of VEGF-A but no significant changes were seen at this dose with either form of VEGF-C. Similarly, no increases in leakage rates over normal wildtype mouse values were found after tail vein injection of $1 \mu \mathrm{g}$ of VEGF-C (TLR: $0.0013 \pm 0.0004$ vs. $0.0011 \pm 0.0005 \mathrm{~min}^{-1}, n=3$ ). We hypothesized that reduced affinity of VEGFR-2 for mature VEGF-C compared to VEGF-A may be an explanation for the lack of effects [31]. After ear injections of a higher dose of $300 \mathrm{ng}$ of VEGF-C, we saw significant increases over PBS injected ears in TLR (2.5-fold, $p<0.001$ ) and VLR (twofold, $p<0.01$ ) when the native form of VEGF-C was injected, but no significant differences in these parameters with the mutant form (Figs. 7b, c). These findings indicate that fully processed VEGF-C can exert leakage effects on blood vessels via activation of VEGFR-2.

Quantification of vascular leakage in subcutaneous $4 \mathrm{~T} 1$ tumors

We next determined if the vascular leakage methods could be adapted for evaluation of deeper tissues, specifically in tumor models where preclinical assessment of the response to anti-angiogenic therapy is critical in the drug development process. We first compared the signal detection of P20-D800 versus ICG and a commonly employed vascular tracer in the visible wavelength spectrum, BSA-Rhodamine [14] through a $1-\mathrm{cm}$ thick chicken breast tissue phantom. As seen in Supplementary Fig. 9, $21 \%$ of signal from P20D800 was detected through the phantom, compared to $18 \%$ of ICG and less than $2 \%$ of BSA-Rhodamine.

Next, we tested the tracers in mice bearing subcutaneous $4 \mathrm{~T} 1$ tumors by performing vascularity and leakage measurements in the tumor versus a normal tissue region of the 

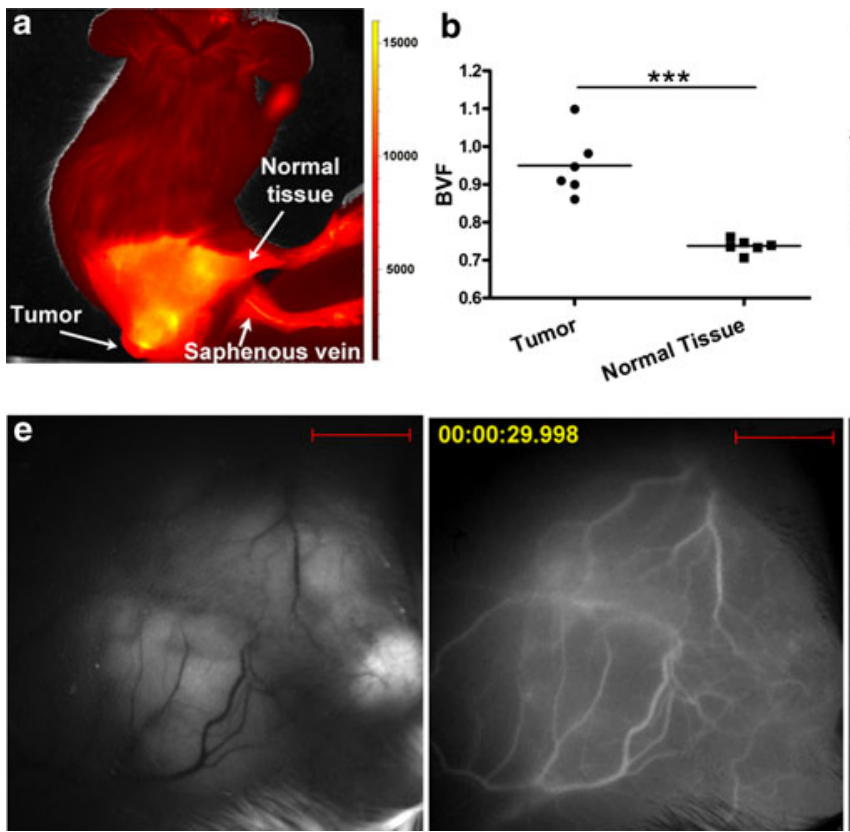

Fig. 8 Measurement of vascularity and leakage in a 4T1 subcutaneous tumor model $(n=6)$. a Positioning of $4 \mathrm{~T} 1$ bearing mice for measurement in IVIS and visualization of increased signal in the 4T1 tumor compared to normal tissue region at $1 \mathrm{~min}$ after intravenous injection of P20-D800. b Quantification of BVF. c TLR. d VLR. e Stereomicroscope imaging of 4T1 tumor blood vessels. The left

same mice after tail vein injection of P20-D800 (Fig. 8a). As seen in Fig. 8b, BVF values were significantly higher (1.3-fold, $p<0.001)$ in the tumor compared to the normal tissue. The vasculature in the tumor region exhibited higher leakage as seen by significant increases in both TLR (threefold, $p<0.001)$ and VLR (2.8-fold, $p<0.001)$ compared to normal tissue (Figs. 8c, d). Higher-resolution dynamic imaging of the tumor associated blood vessels, using a stereomicroscope, also demonstrated significant leakiness after intravenous injection of P20-D680 (Fig. 8e; Supplementary Movie 5).

\section{Discussion}

Regulation of vascular permeability is a critical feature of the microvasculature and alterations in plasma leakage occur in a wide spectrum of conditions. Despite its importance, techniques that can non-invasively quantify vascular leakage in mouse models are quite limited. Established preclinical methods for measuring vascular leakage require either sacrifice or exteriorization of tissue (e.g. Miles assay, FITC-conjugates), or technically demanding and expensive techniques (e.g. MRI, window chambers for in vivo confocal microscopy) $[9,10]$. In this study, we have established and validated imaging methods and quantifications based on visualization of new NIR
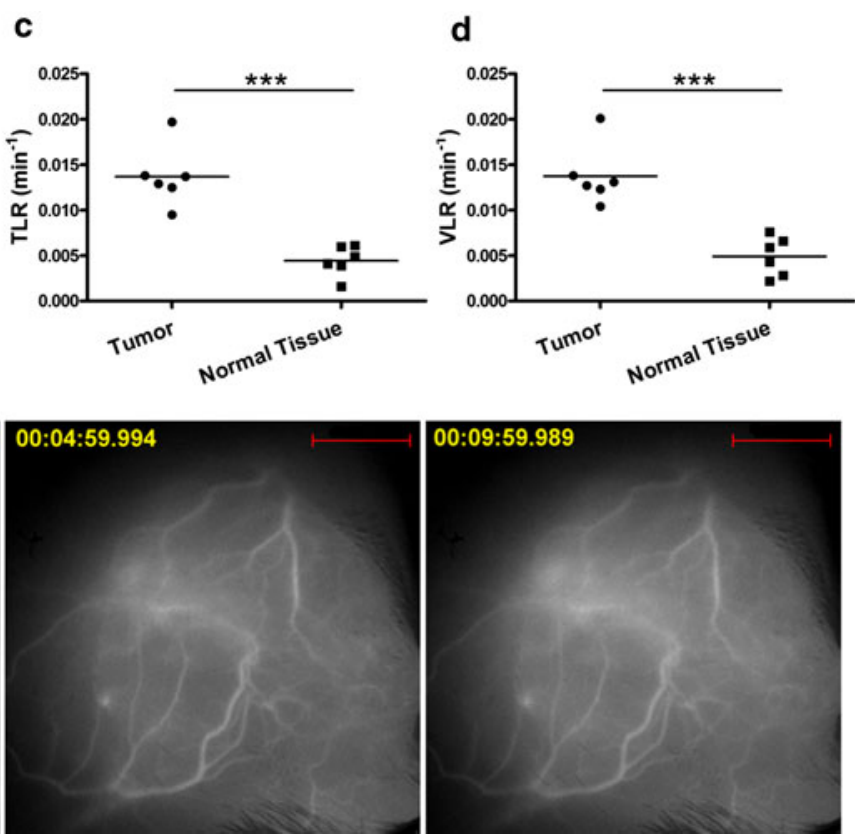

image shows blood vessels and Venus positive tumor fluorescence as seen using a GFP filter set. The three additional panels show imaging with a Cy5 filter set at different time points $(30 \mathrm{~s}, 5 \mathrm{~min}$, and $10 \mathrm{~min}$ ) after intravenous injection of P20-D680 tracers. Note increase in signal outside blood vessels over time, indicating vascular leakage in the tumor. $* p<0.05, * * p<0.01, * * * p<0.001$

tracers that can easily monitor vascular perfusion and leakage within an intact animal, using a commonly available preclinical imaging device. The chosen tracers resulted in an optimal balance between stability in the bloodallowing short-term measurements of leakage-and a clearance compatible with the needs for longitudinal studies.

NIR imaging has great potential for non-invasive imaging due to increased penetration into tissue at these wavelengths. The decreased absorption by water and hemoglobin at these wavelengths and reduced heating effects over visible wavelengths are particularly critical for measurements of vascular leakage. ICG is the most commonly used NIR dye due to its clinical approval and characteristic binding to serum proteins including albumin once injected into the bloodstream [16]. However, we determined that the extremely short half-life of ICG ( $\sim 2 \mathrm{~min}$ ) [32] led to high variability in the serum decay rates between different animals. These features would make it difficult to visualize the leakage of this dye except in cases of extremely leaky vessels. Additionally, ICG has other features that make it unsuitable for accurate and sensitive quantification of vascular leakage, such as dye oligomerization and subsequent self-quenching, and a lack of reactive moieties that hinder its conjugation to other molecules [33, 34]. While liposomal formulations of ICG with improved in vivo stability have been demonstrated for 
both vascular and lymphatic imaging, the size of these particles $(60-200 \mathrm{~nm})$ would severely diminish vascular leakage rates, limiting the quantification potential $[35,36]$.

Therefore, we aimed to develop improved tracers based on a newly developed dye, IRDye 800CW (D800). This dye has several advantages over ICG including increased brightness, ease of conjugation, and long-term stability [34]. Additionally, it has begun a clinical approval process with no adverse effects seen in preclinical studies [34]. However, D800 when injected in its carboxylated form leaks extremely rapidly from blood vessels due to its small size and lack of binding to serum proteins. Therefore, we developed conjugates by binding the amine reactive form of this dye (IRDye 800CW NHS ester) to the hydrophilic polymer PEG, an FDA-approved macromolecule widely used in drug delivery due to its safety profile, low immunogenicity, and ability to prevent protein and cell absorption [37]. The size of these conjugates allowed prolonged circulation lifetime with respect to the free dye and at the same time an effective longitudinal screening due to the good clearance [38]. In addition, we found that the $20 \mathrm{kDa}$ form demonstrated reproducible steady-state vascular leakage into the skin that could be visualized noninvasively in a matter of minutes.

Acquisition of a short dynamic series of NIR images allowed quantification of vascular perfusion and leakage rates in mouse skin with remarkable consistency, as seen by our results analyzing transgenic mice as well as during our longitudinal study. A key advance allowing such reproducible measurements to be developed was normalization of tissue signals to a vascular region of interest, the saphenous vein, which provided adjustments for variations in delivered tracer dose, tissue blood volume fractions, and the dynamics of blood signal. Comparison to the current gold standard, the Miles assay, highlighted the numerous limitations of the existing method as indicated by previous investigators [8]. Although both methods were able to demonstrate significantly increased leakage in response to VEGF-A injections into the skin, the Miles assay gave only information on total tissue concentration of the dye at $30 \mathrm{~min}$ after injection and required sacrifice of the animal. In situations of increased vascularity, such as in the VEGFA transgenic animals or under inflammatory conditions, the Miles assay would be even more limited because it cannot provide adjustments for increased vascular surface area. Both methods, however, are not designed to distinguish between intercellular and transcellular routes of vascular leakage.

Although intradermal injections are the most widely used route to test permeability factors, we found high variability in our measurements, even in the saline control group. It is likely that direct injections into the skin can rupture blood vessels and/or cause increases in hydraulic conductivity that can increase transport of tracers from plasma through the interstitium. When the protein was injected intravascularly along with the tracer, we were able to quantify leakage effects much more consistently. By limiting the technical variability in making measurements of vascular leakage, we were able to determine highly significant differences with even small numbers of mice. Additionally, the ability to accurately image repeatedly in the same cohorts of mice gives researchers a powerful tool that can reduce the effects of inherent biological variability by providing an intra-individual baseline measurement in longitudinal studies of therapeutic interventions, potentially reducing the number of animals needed for such studies. As permeability changes occur quite rapidly in response to drug interventions, sensitive in vivo readouts of vascular leakage could represent a major step forward in the preclinical phase of drug development.

The increases in vascular leakage in K14-VEGF-C overexpressing mice after VEGF-A was administered may highlight an important compensatory role of a functional lymphatic system during conditions of hyperpermeability. The K14-VEGF-C mice have an expanded network of dermal lymphatics and enhanced lymphatic function, as shown by increased clearance of the tracers after extravasation or after skin injection of Evans Blue dye [27]. The hyperplastic lymphatic capillaries in these animals were previously suggested to be less functional $[17,26]$. However, the increased surface area available for absorption may accelerate lymphatic clearance and together with increased vascular leakage rates, result in enhanced "tissue flushing." Recent data from our lab and others indicate a key role for functional lymphatic vessels induced by VEGF-C overexpression in limiting inflammation and accelerating diabetic wound healing [27, 39-41]. An expanded lymphatic network may be more primed to deal with an inflammatory insult by limiting interstitial fluid pressure and the resultant tissue swelling. This increased "edema safety factor" may partly explain the lack of regression of newly formed lymphatic vessels after resolution of chronic inflammation [42, 43].

Although the connection of VEGF-A and angiogenic vessels to increased vascular permeability is well established, the relationship between the functionality of the lymphatic system and vascular leakage has not been extensively studied. Dysfunctional lymphatic function has been speculated as part of the reason for the enhanced permeability and retention effect that aids delivery of nanoparticles to tumors [44]. However, in contrast to this, the high interstitial pressure that can result from chronic hyperpermeability and poor lymphatic function has also been considered as a barrier to drug delivery [45]. As the lymphatic network represents the only exit route from the interstitium for macromolecules, an improved or 
diminished function of this system likely will also have an effect on the rate of capillary filtration as predicted by the revised Starling equation [1, 3]. Thus, VEGF-C might enhance vascular leakage by two different mechanismsdirect effects of the processed form on blood vasculature endothelium via activation of VEGFR2, as shown by previous studies [46, 47] and in our intradermal injection experiments, and indirect effects via establishment of a more efficient lymphatic drainage network. Although more work is necessary to validate this hypothesis, our findings may hint at strategies to improve lymphatic function to supplement existing therapies and enhance the resolution of chronic inflammatory disorders. Tools that can evaluate blood volume fraction and vascular leakage rates such as developed in the current study would allow for improved monitoring of drug penetration and response in these conditions.

We have successfully applied the noninvasive measurements of blood volume fraction and vascular leakage to a subcutaneous 4T1 tumor model. The increased depth penetration of NIR tracers allows for measurement of tissues at least $1 \mathrm{~cm}$ deep, as evidenced by tissue phantom experiments and our previous experience of deep iliac lymph node detection in mice with ICG liposomes [36]. When we performed measurements in the $4 \mathrm{~T} 1$ tumor, we were able to detect significantly increased vascularity and leakage rates compared to normal tissue, thus enabling future studies to evaluate the effects of anti-angiogenic therapies on these parameters which might facilitate the drug development process.

Acknowledgments The authors would like to thank Carlos Ochoa for assistance with animal care and Sinem Karaman and Alexandra Ochsenbein for technical assistance. This work was supported by a Whitaker International Scholar grant (to S.T.P.); National Institutes of Health grant CA69184, Swiss National Science Foundation grants 3100A0-108207 and 31003A-130627, Commission of the European Communities grant LSHC-CT-2005-518178, Advanced European Research Council grant LYVICAM, Oncosuisse and Krebsliga Zurich (to M.D.).

Conflict of interest The authors declare that they have no conflict of interest.

\section{References}

1. Yuan SY, Rigor RR (2010) In: Regulation of Endothelial Barrier Function. Integrated Systems Physiology: From Molecule to Function to Disease. San Rafael,CA

2. Levick JR, Michel CC (2010) Microvascular fluid exchange and the revised Starling principle. Cardiovasc Res 87(2):198-210. doi: $10.1093 / \mathrm{cvr} / \mathrm{cvq} 062$

3. Scallan J, Huxley VH, Korthuis RJ (2010). In: Capillary fluid exchange: regulation, functions, and pathology. Integrated systems physiology: from molecule to function to disease. San Rafael, CA
4. Nagy JA, Dvorak AM, Dvorak HF (2007) VEGF-A and the induction of pathological angiogenesis. Annu Rev Pathol 2:251-275. doi:10.1146/annurev.pathol.2.010506.134925

5. Dejana E, Tournier-Lasserve E, Weinstein BM (2009) The control of vascular integrity by endothelial cell junctions: molecular basis and pathological implications. Dev Cell 16(2):209-221. doi: 10.1016/j.devcel.2009.01.004

6. Miles AA, Miles EM (1952) Vascular reactions to histamine, histamine-liberator and leukotaxine in the skin of guinea-pigs. J Physiol 118(2):228-257

7. Nagy JA, Benjamin L, Zeng H, Dvorak AM, Dvorak HF (2008) Vascular permeability, vascular hyperpermeability and angiogenesis. Angiogenesis 11(2):109-119. doi:10.1007/s10456-0089099-z

8. Bates DO (2010) Vascular endothelial growth factors and vascular permeability. Cardiovasc Res 87(2):262-271. doi: 10.1093/cvr/cvq105

9. Curry FR, Adamson RH (2010) Vascular permeability modulation at the cell, microvessel, or whole organ level: towards closing gaps in our knowledge. Cardiovasc Res 87(2):218-229. doi:10.1093/cvr/cvq115

10. Kenne E, Lindbom L (2011) Imaging inflammatory plasma leakage in vivo. Thromb Haemost 105(5):783-789. doi:10.1160/ TH10-10-0635

11. Neeman M, Dafni H (2003) Structural, functional, and molecular MR imaging of the microvasculature. Annu Rev Biomed Eng 5:29-56. doi:10.1146/annurev.bioeng.5.040202.121606

12. Mulder WJ, Griffioen AW (2010) Imaging of angiogenesis. Angiogenesis 13(2):71-74. doi:10.1007/s10456-010-9178-9

13. Dreher MR, Liu W, Michelich CR, Dewhirst MW, Yuan F, Chilkoti A (2006) Tumor vascular permeability, accumulation, and penetration of macromolecular drug carriers. J Natl Cancer Inst 98(5):335-344. doi:10.1093/jnci/djj070

14. Vandoorne K, Addadi Y, Neeman M (2010) Visualizing vascular permeability and lymphatic drainage using labeled serum albumin. Angiogenesis 13(2):75-85. doi:10.1007/s10456-010-9170-4

15. Hilderbrand SA, Weissleder R (2010) Near-infrared fluorescence: application to in vivo molecular imaging. Curr Opin Chem Biol 14(1):71-79. doi:10.1016/j.cbpa.2009.09.029

16. Cherrick GR, Stein SW, Leevy CM, Davidson CS (1960) Indocyanine green: observations on its physical properties, plasma decay, and hepatic extraction. J Clin Invest 39:592-600. doi: 10.1172/JCI104072

17. Jeltsch M, Kaipainen A, Joukov V, Meng X, Lakso M, Rauvala H, Swartz M, Fukumura D, Jain RK, Alitalo K (1997) Hyperplasia of lymphatic vessels in VEGF-C transgenic mice. Science 276(5317):1423-1425

18. Xia YP, Li B, Hylton D, Detmar M, Yancopoulos GD, Rudge JS (2003) Transgenic delivery of VEGF to mouse skin leads to an inflammatory condition resembling human psoriasis. Blood 102(1):161-168. doi:10.1182/blood-2002-12-3793

19. Thurston G, Suri C, Smith K, McClain J, Sato TN, Yancopoulos GD, McDonald DM (1999) Leakage-resistant blood vessels in mice transgenically overexpressing angiopoietin- 1 . Science 286(5449):2511-2514

20. Wyatt SK, Manning HC, Bai M, Bailey SN, Gallant P, Ma G, McIntosh L, Bornhop DJ (2010) Molecular imaging of the translocator protein (TSPO) in a pre-clinical model of breast cancer. Mol Imaging Biol 12(3):349-358. doi:10.1007/s11307-009-0270-8

21. Ott P, Keiding S, Bass L (1993) Plasma elimination of indocyanine green in the intact pig after bolus injection and during constant infusion: comparison of spectrophotometry and highpressure liquid chromatography for concentration analysis. Hepatology 18(6):1504-1515. doi:10.1002/hep.1840180633

22. Kunstfeld R, Hirakawa S, Hong YK, Schacht V, Lange-Asschenfeldt B, Velasco P, Lin C, Fiebiger E, Wei X, Wu Y, Hicklin 
D, Bohlen P, Detmar M (2004) Induction of cutaneous delayedtype hypersensitivity reactions in VEGF-A transgenic mice results in chronic skin inflammation associated with persistent lymphatic hyperplasia. Blood 104(4):1048-1057. doi: 10.1182/blood-2003-08-2964

23. Detmar M, Brown LF, Schon MP, Elicker BM, Velasco P, Richard L, Fukumura D, Monsky W, Claffey KP, Jain RK (1998) Increased microvascular density and enhanced leukocyte rolling and adhesion in the skin of VEGF transgenic mice. J Invest Dermatol 111(1):1-6. doi:10.1046/j.1523-1747.1998.00262.x

24. Halin C, Detmar M (2008) Chapter 1. Inflammation, angiogenesis, and lymphangiogenesis. Methods Enzymol 445:1-25. doi: 10.1016/S0076-6879(08)03001-2

25. Dafni H, Landsman L, Schechter B, Kohen F, Neeman M (2002) MRI and fluorescence microscopy of the acute vascular response to VEGF165: vasodilation, hyper-permeability and lymphatic uptake, followed by rapid inactivation of the growth factor. NMR Biomed 15(2):120-131

26. Lohela M, Helotera H, Haiko P, Dumont DJ, Alitalo K (2008) Transgenic induction of vascular endothelial growth factor-C is strongly angiogenic in mouse embryos but leads to persistent lymphatic hyperplasia in adult tissues. Am J Pathol 173(6): 1891-1901. doi:10.2353/ajpath.2008.080378

27. Huggenberger R, Siddiqui SS, Brander D, Ullmann S, Zimmermann K, Antsiferova M, Werner S, Alitalo K, Detmar M (2011) An important role of lymphatic vessel activation in limiting acute inflammation. Blood 117(17):4667-4678. doi:10.1182/blood2010-10-316356

28. Krynyckyi BR, Kim CK, Goyenechea MR, Chan PT, Zhang ZY, Machac J (2004) Clinical breast lymphoscintigraphy: optimal techniques for performing studies, image atlas, and analysis of images. Radiographics 24 (1):121-145; discussion 139-145. doi: 10.1148/rg.241025713

29. Karlsen TV, McCormack E, Mujic M, Tenstad O, Wiig H (2011) Minimally-invasive quantification of lymph flow in mice and rats by imaging depot clearance of near-infrared albumin. Am J Physiol Heart Circ Physiol. doi:10.1152/ajpheart.00842.2011

30. Tammela T, Alitalo K (2010) Lymphangiogenesis: molecular mechanisms and future promise. Cell 140(4):460-476. doi: 10.1016/j.cell.2010.01.045

31. Joukov V, Kumar V, Sorsa T, Arighi E, Weich H, Saksela O, Alitalo K (1998) A recombinant mutant vascular endothelial growth factor-C that has lost vascular endothelial growth factor receptor-2 binding, activation, and vascular permeability activities. J Biol Chem 273(12):6599-6602

32. Saxena V, Sadoqi M, Shao J (2003) Degradation kinetics of indocyanine green in aqueous solution. J Pharm Sci 92(10): 2090-2097. doi:10.1002/jps. 10470

33. Troyan SL, Kianzad V, Gibbs-Strauss SL, Gioux S, Matsui A, Oketokoun R, Ngo L, Khamene A, Azar F, Frangioni JV (2009) The FLARE intraoperative near-infrared fluorescence imaging system: a first-in-human clinical trial in breast cancer sentinel lymph node mapping. Ann Surg Oncol 16(10):2943-2952. doi: 10.1245/s10434-009-0594-2

34. Marshall MV, Draney D, Sevick-Muraca EM, Olive DM (2010) Single-dose intravenous toxicity study of IRDye $800 \mathrm{CW}$ in Sprague-Dawley rats. Mol Imaging Biol 12(6):583-594. doi: 10.1007/s11307-010-0317-x
35. Sandanaraj BS, Gremlich HU, Kneuer R, Dawson J, Wacha S (2010) Fluorescent nanoprobes as a biomarker for increased vascular permeability: implications in diagnosis and treatment of cancer and inflammation. Bioconjug Chem 21(1):93-101. doi: $10.1021 / \mathrm{bc} 900311 \mathrm{~h}$

36. Proulx ST, Luciani P, Derzsi S, Rinderknecht M, Mumprecht V, Leroux JC, Detmar M (2010) Quantitative imaging of lymphatic function with liposomal indocyanine green. Cancer Res 70(18):7053-7062. doi:10.1158/0008-5472.CAN-10-0271

37. Greenwald RB, Choe YH, McGuire J, Conover CD (2003) Effective drug delivery by PEGylated drug conjugates. Adv Drug Deliv Rev 55(2):217-250

38. Fox ME, Szoka FC, Frechet JM (2009) Soluble polymer carriers for the treatment of cancer: the importance of molecular architecture. Acc Chem Res 42(8):1141-1151. doi:10.1021/ar900035f

39. Huggenberger R, Ullmann S, Proulx ST, Pytowski B, Alitalo K, Detmar M (2010) Stimulation of lymphangiogenesis via VEGFR3 inhibits chronic skin inflammation. J Exp Med 207(10): 2255-2269. doi:10.1084/jem.20100559

40. Zhou Q, Guo R, Wood R, Boyce BF, Liang Q, Wang YJ, Schwarz EM, Xing L (2011) Vascular endothelial growth factor C attenuates joint damage in chronic inflammatory arthritis by accelerating local lymphatic drainage in mice. Arthritis Rheum 63(8):2318-2328. doi:10.1002/art.30421

41. Saaristo A, Tammela T, Farkkila A, Karkkainen M, Suominen E, Yla-Herttuala S, Alitalo K (2006) Vascular endothelial growth factor-C accelerates diabetic wound healing. Am J Pathol 169(3):1080-1087. doi:10.2353/ajpath.2006.051251

42. Baluk P, Tammela T, Ator E, Lyubynska N, Achen MG, Hicklin DJ, Jeltsch M, Petrova TV, Pytowski B, Stacker SA, Yla-Herttuala S, Jackson DG, Alitalo K, McDonald DM (2005) Pathogenesis of persistent lymphatic vessel hyperplasia in chronic airway inflammation. J Clin Invest 115(2):247-257. doi:10.1172/ JCI22037

43. Proulx ST, Kwok E, You Z, Beck CA, Shealy DJ, Ritchlin CT, Boyce BF, Xing L, Schwarz EM (2007) MRI and quantification of draining lymph node function in inflammatory arthritis. Ann N Y Acad Sci 1117:106-123. doi:10.1196/annals.1402.016

44. Greish K (2007) Enhanced permeability and retention of macromolecular drugs in solid tumors: a royal gate for targeted anticancer nanomedicines. J Drug Target 15(7-8):457-464. doi: $10.1080 / 10611860701539584$

45. Jain RK, Stylianopoulos T (2010) Delivering nanomedicine to solid tumors. Nat Rev Clin Oncol 7(11):653-664. doi:10.1038/ nrclinonc.2010.139

46. Witzenbichler B, Asahara T, Murohara T, Silver M, Spyridopoulos I, Magner M, Principe N, Kearney M, Hu JS, Isner JM (1998) Vascular endothelial growth factor-C (VEGF-C/VEGF-2) promotes angiogenesis in the setting of tissue ischemia. Am J Pathol 153(2):381-394. doi:10.1016/S0002-9440(10)65582-4

47. Saaristo A, Veikkola T, Enholm B, Hytonen M, Arola J, Pajusola K, Turunen P, Jeltsch M, Karkkainen MJ, Kerjaschki D, Bueler H, Yla-Herttuala S, Alitalo K (2002) Adenoviral VEGF-C overexpression induces blood vessel enlargement, tortuosity, and leakiness but no sprouting angiogenesis in the skin or mucous membranes. FASEB J 16(9):1041-1049. doi:10.1096/fj.01-1042com 